\title{
Acousto-Optic Modulator Driven by Surface Acoustic Waves
}

\author{
S. KAKIO* \\ Interdisciplinary Graduate School of Medicine and Engineering, University of Yamanashi, Kofu 400-8511, Japan
}

\begin{abstract}
In this paper, an introduction to the design, fabrication, evaluation, and application of acousto-optic modulators using the Bragg diffraction of an optical guided wave in a channel optical waveguide due to a surface acoustic wave is provided. First, the surface acoustic wave mode dependence in the acousto-optic Bragg diffraction was discussed by considering the surface acoustic wave power required for the maximum diffraction $P_{100}$ calculated using coupled mode theory and the measured $P_{100}$ in Ti-diffused planar optical waveguides fabricated on $\mathrm{LiNbO}_{3}$ substrates. Next, performances of several devices that the author and the coworkers developed are reviewed. These include a waveguide-type acousto-optic modulator using a tapered crossed-channel proton-exchanged optical waveguide on a $128^{\circ}$-rotated $Y$-cut $\mathrm{LiNbO}_{3}$ substrate for an optical wavelength of $1.55 \mu \mathrm{m}$, a monolithically integrated tandem acousto-optic modulator in which several waveguide-type acousto-optic modulators are connected in tandem on the same substrate, and a waveguide-type acousto-optic modulator in which laser lights of the three primary colors, red, green, and blue, can be modulated by the same modulator at the same driving frequency.
\end{abstract}

DOI: 10.12693 /APhysPolA.127.15

PACS: 42.79.Jq, 43.35.Sx, 78.20.Hp, 78.20.hb

\section{Introduction}

Acousto-optic modulators (AOMs) using the Bragg diffraction have been widely used as optical control devices such as optical switches, optical deflectors, optical frequency shifters, and so on, for a long time. In general, a lower driving power is needed for their applications. A typical AOM is a bulk-type AOM, in which the incident light is diffracted by a bulk acoustic wave through a photoelastic effect. In this case, the material of the bulk crystal does not necessarily have to be piezoelectric because the bulk acoustic wave is launched from the transducer bonded to the crystal. Therefore, a bulk crystal with a large figure of merit (FOM), such as $\mathrm{TeO}_{2}$ or $\mathrm{As}_{2} \mathrm{~S}_{3}$ can be used.

On the other hand, for an AOM driven by a surface acoustic wave (SAW), earlier studies suggested a structure consisting of a thin film deposited on a piezoelectric substrate, such as $\mathrm{LiNbO}_{3}$ (LN) [1]. The thin film acts as a planar optical waveguide, and the optical guided wave in the thin film is diffracted by the SAW launched from an interdigital transducer (IDT). In this case, too, a material with a large FOM can be used as the thin film. In 1982, Tsai reported such an AOM driven by a SAW using optical guided waves in a Ti-diffused crossedchannel waveguide on $Y$-cut LN. In this waveguide-type AOM, the channel waveguides were crossed at the Bragg angle on the substrate [2]. A diffraction efficiency of $70 \%$ was obtained at a SAW power of $300 \mathrm{~mW}$ and an optical wavelength of $0.633 \mu \mathrm{m}$. Compared with a bulk-type AOM and a planar waveguide-type AOM, such a configuration is more suitable for integrating and stabilizing the modulation system. In addition, a direct connection to an optical fiber is also possible. In a waveguide-type

*e-mail: kakio@yamanashi.ac.jp
AOM driven by a SAW, the requirements for the substrate are a low-loss optical waveguide, a high electromechanical coupling factor, and a large photoelastic constant. Almost the only material satisfying these requirements is LN. However, the FOM of LN is smaller than that of $\mathrm{As}_{2} \mathrm{~S}_{3}$ [3]. Therefore, a suitable configuration and parameters are important to obtain a lower driving power.

In this paper, first, the SAW mode (a Rayleigh wave or a leaky SAW) dependence in the AO Bragg diffraction was discussed. Next, performances of several devices that the author and the coworkers developed [4-12] are reviewed.

\section{SAW mode dependence in AO Bragg diffraction}

The typical SAW propagation mode is a Rayleigh wave with longitudinally and vertically polarized shear particle motion. The Rayleigh wave has been utilized in AO devices driven by an SAW, and conditions for obtaining a lower driving power in planer guided-wave AO Bragg diffraction, such as TE-, TM-mode coupling to the SAW, optical wavelength dependence, and optimum SAW frequency have been clarified [13-15]. On the other hand, a leaky SAW (LSAW), also called a shear-horizontal-type SAW (SH-SAW), mainly consists of horizontally polarized shear particle motion. By utilizing an LSAW, a lower driving power in planer guided-wave AO Bragg diffraction can be expected because the LSAW generally has a higher electromechanical coupling factor $\left(K^{2}\right)$ than the Rayleigh wave. However, little is known about the experimental investigation. To investigate this, two substrates were chosen, one was $128^{\circ} Y$-LN for the Rayleigh wave, and the other was $64^{\circ}$-rotated $Y$-cut LN ( $64^{\circ} Y$-LN) for the LSAW, because these substrates have a high $K^{2}$ for each SAW mode [16].

A Ti-diffused planar waveguide with a depth $d$ of approximately $2 \mu \mathrm{m}$ and interdigital transducers (IDTs) with a period $\Lambda$ of $20 \mu \mathrm{m}, 20$ finger pairs, and an over- 
lap length $L_{\mathrm{g}}$ of $3 \mathrm{~mm}$ were fabricated on each substrate. A He-Ne laser light with a wavelength $\lambda$ of $0.633 \mu \mathrm{m}$ was guided to the Ti-diffused waveguide using the rutile prism coupler, and a RF voltage with approximately $200 \mathrm{MHz}$ was applied to the input IDT. The output light intensity was measured using a photodetector. Diffraction efficiency was determined from the decrease in the intensity of the undiffracted light. The SAW power in the interaction region was determined from the ratio of the vertical particle displacement on the surface measured by an optical probe method to the calculated one.

The measured diffraction efficiency as a function of the square root of the SAW power is shown in Fig. 1. The measured results were fitted using the $\sin ^{2}$-curve solution of coupled-mode equations [13]. The SAW power required for maximum diffraction $P_{100}$ was determined from the peak power of the fitted curve. Maximum diffraction efficiency for $\mathrm{Ti} / 64^{\circ} Y$-LN was not obtained experimentally. The maximum diffraction efficiency of $85 \%$ measured in $\mathrm{TM}_{0}$ mode of $\mathrm{Ti} / 128^{\circ} Y$-LN was assumed. The experimental values of $P_{100}$ of $\mathrm{TM}_{0}$ mode were determined to be $0.06 \mathrm{~W}$ for $\mathrm{Ti} / 128^{\circ} Y$-LN and $400 \mathrm{~W}$ for $\mathrm{Ti} / 64^{\circ} Y$-LN, respectively. The $P_{100}$ for the LSAW is approximately $10^{4}$ times larger than that for the Rayleigh wave.
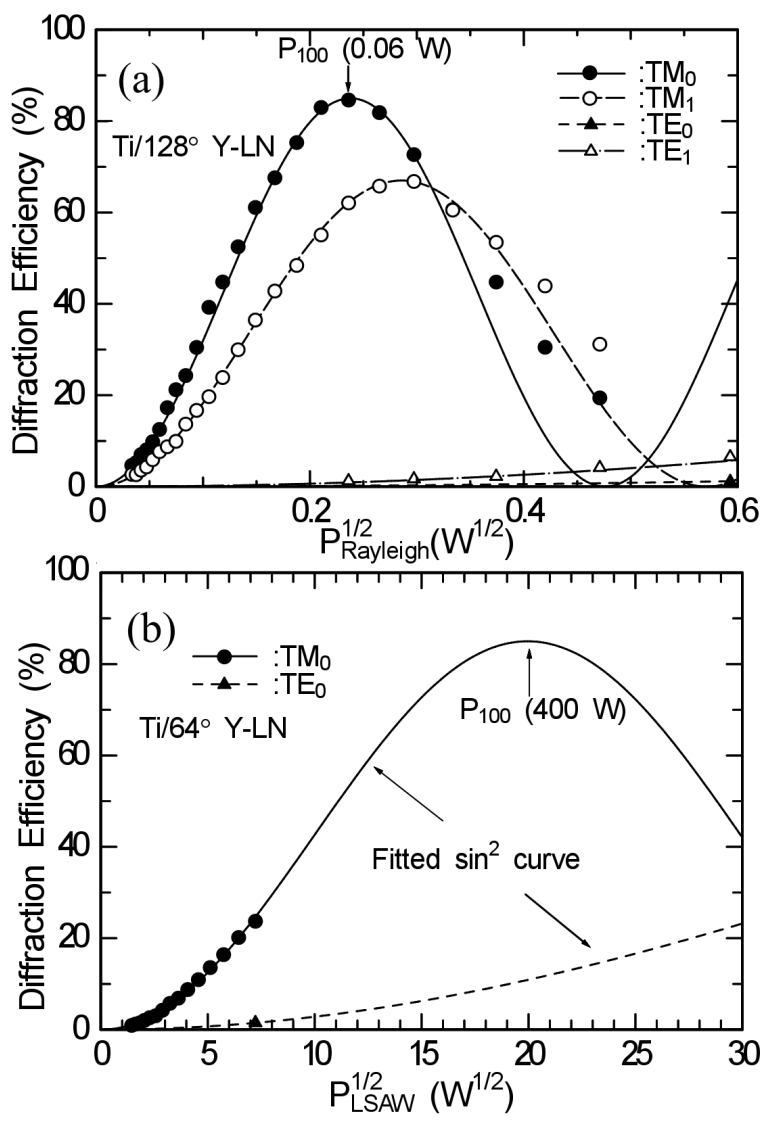

Fig. 1. Measured diffraction efficiency for (a) Ti/128 $Y$-LN and (b) $\mathrm{Ti} / 64^{\circ} Y$-LN samples.

The theoretical $P_{100}$ for $\mathrm{TM}_{0}$ mode was calculated by using the coupled mode theory [13]. $P_{100}$ is obtained by the relationship

$$
P_{100}=\left(\frac{\pi}{2\left|C_{21}\right|}\right)^{2} \frac{1}{L}(\mathrm{~W}),
$$

where $L$ is the length of the interaction region and $C_{21}$ is a coupling coefficient expressed by

$$
C_{21}=\frac{1}{480 \Lambda} \int_{-\infty}^{d}\left(E_{2}^{\mathrm{i}} \Delta \varepsilon_{22} E_{2}^{\mathrm{d} *}+E_{3}^{\mathrm{i}} \Delta \varepsilon_{33} E_{3}^{\mathrm{d} *}\right) \mathrm{d} x_{3} .
$$

The overlap integral in the depth direction $x_{3}$ between the electric field of the incident $\left(E_{2}^{\mathrm{i}}, E_{3}^{\mathrm{i}}\right)$ and diffracted optical guide waves $\left(E_{2}^{\mathrm{d}}, E_{3}^{\mathrm{d}}\right)$ and the perturbations on the dielectric tensor, $\Delta \varepsilon_{22}$ and $\Delta \varepsilon_{33}$, caused by the strain of the LSAW was calculated from a depth of $10 \Lambda$ to the surface because the LSAW does not decay to zero in the depth direction. Figure $2 \mathrm{a}$ and $\mathrm{b}$ shows the real parts of the calculated $\Delta \varepsilon_{22}$ and $\Delta \varepsilon_{33}$ in the depth direction for each SAW mode when the depth of Ti-diffused layer is 0.11 . The LSAW has a larger $\Delta \varepsilon_{22}$ than that for the Rayleigh wave. However, for the primary perturbation $\Delta \varepsilon_{33}$ for Bragg diffraction, it can be seen that the LSAW has a smaller $\Delta \varepsilon_{33}$ than that for the Rayleigh wave.

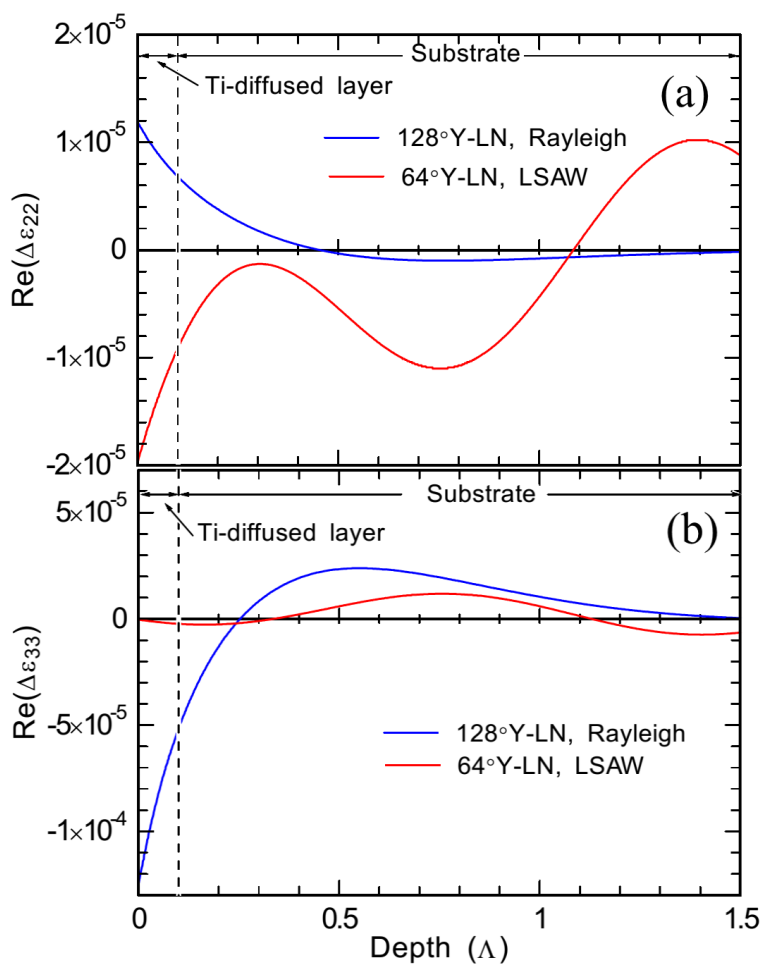

Fig. 2. Real parts of the calculated perturbations on the dielectric tensor in the depth direction, (a) $\Delta \varepsilon_{22}$ and (b) $\Delta \varepsilon_{33}$.

Figure 3 shows the theoretical $P_{100}$ as functions of the normalized waveguide depth $d / \Lambda$. The theoretical values were close to the experimental values. Moreover, for a TE-mode light beam, $10-10^{3}$ times larger driving power than that for a TM-mode light beam was required for the Bragg diffraction for both cases of the LSAW and the Rayleigh wave. Therefore, it was confirmed that 


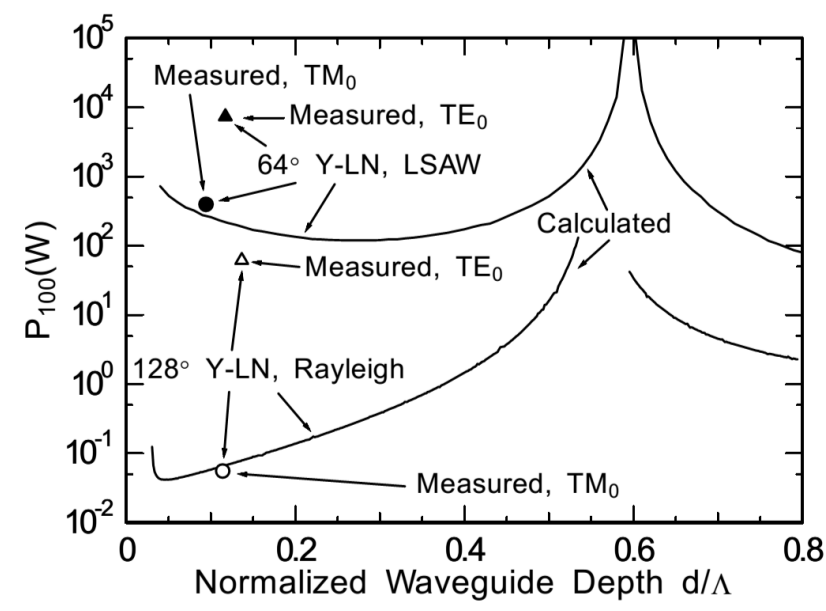

Fig. 3. SAW power required for $100 \%$ diffraction. $\mathrm{Ti} /$ rotated $Y$-cut $\mathrm{LiNbO}_{3}, \lambda=0.633 \mu \mathrm{m}, \Lambda=20 \mu \mathrm{m}$.

a suitable combination for the Bragg diffraction is the TM mode with a Rayleigh wave. For a configuration for polarization control of the optical guided wave by utilizing an LSAW, comparable perturbations to those in conventional Bragg diffraction due to a Rayleigh wave was reported [17].

\section{Waveguide-type AOM for optical wavelength of $1.55 \mu \mathrm{m}$}

For an optical wavelength of $1.55 \mu \mathrm{m} \quad[4-7]$ a waveguide-type AOM with a combination of the TM mode and a Rayleigh wave was developed. The configuration of the waveguide-type AOM is shown in Fig. 4. The channel waveguide was fabricated in the $128^{\circ} \mathrm{Y}$ LN substrate using the proton exchange (PE) method and post-annealing, which is more easily realized because of the low-temperature process. The Rayleigh wave is launched from the IDT fabricated on the substrate to the diffraction region.

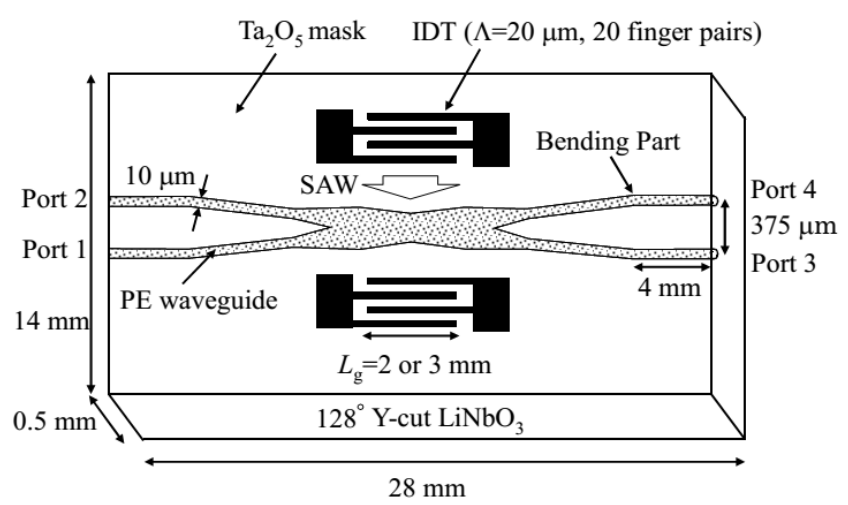

Fig. 4. Configuration of waveguide-type AOM [6].

Figure 5 shows the waveguide shape in the diffraction region of the waveguide-type AOM. The $P_{100}$ is in inverse proportion to the length of the interaction region. To increase the length of the interaction region, $10 \mu \mathrm{m}$ width of the input and output waveguides is increased

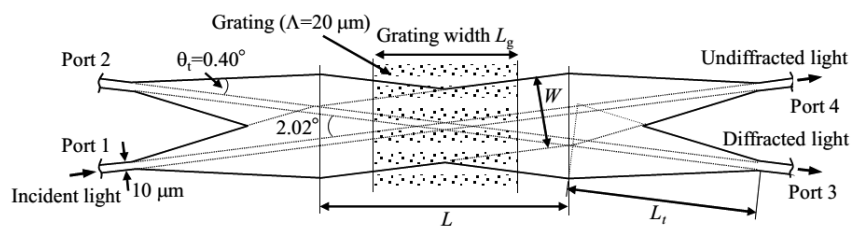

Fig. 5. Waveguide shape in diffraction region of waveguide-type AOM [7].

to a width $W$ of $120-150 \mu \mathrm{m}$ using the tapered waveguides with a length of $L_{t}$ of $7.5-10 \mathrm{~mm}$. The incident light from Port 1 is diffracted by a SAW in the area of SAW propagation and guided to Port 3, with a Bragg angle of $1.01^{\circ}$, given by

$$
\theta_{\mathrm{B}}=\sin ^{-1} \frac{\lambda}{2 N \Lambda}
$$

where $N$ is the effective refractive index. The diffracted light is corrected by a tapered waveguide and guided to the waveguide of Port 3 . The waveguide shape was designed using beam-propagation analysis.

The channel waveguide was fabricated by first forming an RF-sputtered $\mathrm{Ta}_{2} \mathrm{O}_{5}$ mask with a film thickness of $0.08 \mu \mathrm{m}$ by the lift-off method on $128^{\circ} Y$-LN, and then by the PE process and post-annealing. A PE time was $1 \mathrm{~h} 15 \mathrm{~min}$ in a solution of benzoic acid containing $0.2 \mathrm{~mol} \%$ lithium benzoate at $240^{\circ} \mathrm{C}$, and an annealing time was $1 \mathrm{~h} 40 \mathrm{~min}$ at $400^{\circ} \mathrm{C}$. A Gaussian index profile with a change in extraordinary refractive index $\left(\Delta n_{\mathrm{e}}\right)$ of 0.021 and a waveguide depth of $4.1 \mu \mathrm{m}$ at $\Delta n_{\mathrm{e}} / \mathrm{e}$ $(\mathrm{e}=2.718 . .$.$) was obtained. After polishing and AR-$ coating the end face of the waveguide, a pair of normal IDT with $\Lambda=20 \mu \mathrm{m}$ and an overlap length of $L_{\mathrm{g}}$ were formed on the substrate by an aluminum thin film.

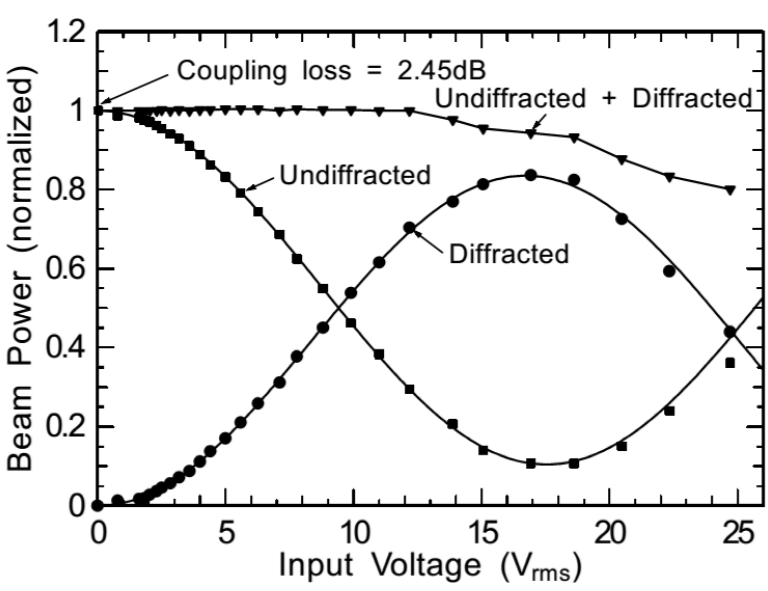

Fig. 6. Measured diffraction efficiency for samples with $L=4 \mathrm{~mm}$ and $L_{\mathrm{g}}=2 \mathrm{~mm}$ [7].

The diffraction properties of the waveguide-type AOM were measured using a $1.55 \mu \mathrm{m}$ laser diode. Figure 6 shows an example of the measured diffraction property for a sample with $L=4 \mathrm{~mm}$ and $L_{\mathrm{g}}=2 \mathrm{~mm}$ at a driving frequency of $195 \mathrm{MHz}$. An $84 \%$ diffraction efficiency was obtained at an input voltage of $17 \mathrm{~V}_{\mathrm{rms}}$. 


\section{Monolithically integrated tandem AOM}

The optical frequency of diffracted light is Doppler shifted by the driving frequency in an AOM. This is one of unique advantages of the AO switches over the electrooptic (EO) switches, although the speed of the AO switches is much slower than the EO switches. However, the frequency shift of a simple AOM is almost fixed to around the center frequency of the transducer. Tsai et al. proposed the integrated AO devices in which several planar guided-wave AO Bragg deflectors are connected on the same substrate to realize frequency shifter and space switch [18-20]. In this section, a structure in which several waveguide-type AOMs are connected in tandem on the same substrate was proposed [8-10].

The overall configuration of the monolithically integrated tandem waveguide-type AOM, hereafter abbreviated as the tandem AOM, is shown in Fig. 7. The two output ports of the first-stage 2-by-2 switch are connected to the input ports of the two second-stage 1-by-2 switches on the same substrate. A $100 \mu \mathrm{m}$ wide straight waveguide was used to connect these switches. Two pairs of IDTs with $\Lambda=32 \mu \mathrm{m}$ and an overlap length of $2 \mathrm{~mm}$ were used to propagate the Rayleigh wave in the first and second stages. The optical frequency shifts by the sum of the two driving frequencies, and the difference between them can be obtained by combining the upward and downward frequency shifts in the first and second SAWs. The waveguide shape of the tandem AOM was also designed using beam propagation analysis, and the tandem AOM was fabricated using a similar process to the single AOM. The AOM was incorporated into a module and optically connected to input and output polarizationmaintained (PM) fiber arrays through collimating and condensing lenses. Figure 8 shows a photograph of the module. The module device is in trial production in a cooperative company.

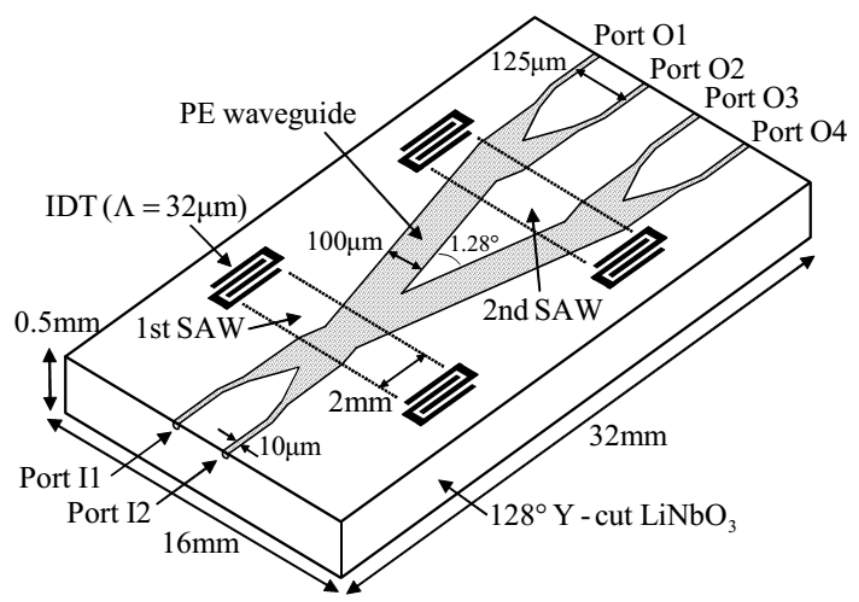

Fig. 7. Configuration of monolithically integrated tandem waveguide-type AOM [8].

Diffraction properties were measured using a $1.55 \mu \mathrm{m}$ laser diode at a driving frequency of approximately $120 \mathrm{MHz}$. Maximum diffraction efficiencies of

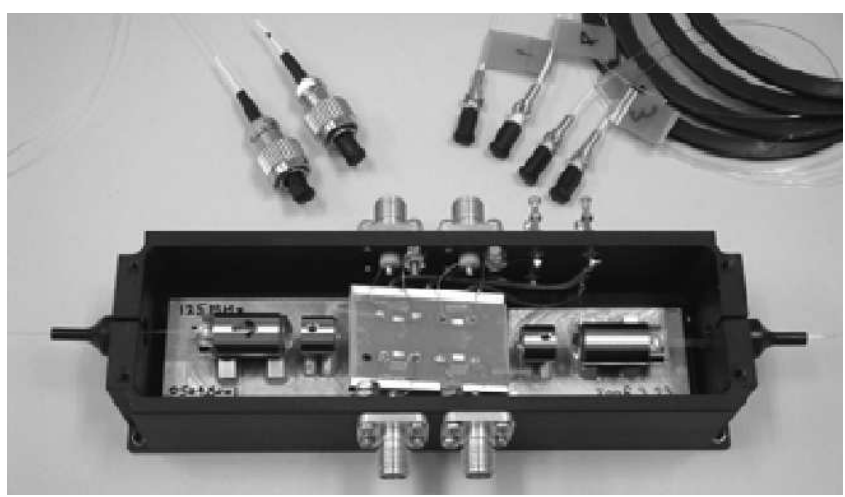

Fig. 8. Photograph of tandem AOM module [8].

$92 \%$ and $83 \%$ were obtained using the first and second SAWs, respectively. When the first SAW was fixed at the maximum diffraction efficiency and the input voltage supplied to the second SAW was increased, a peak diffraction efficiency of $63 \%$ was obtained at an input voltage of $11 \mathrm{~V}_{\mathrm{rms}}$ at the same frequency.

By using the tandem AOM, the optical frequency shifts for the sum of two driving frequencies and the difference frequency ranging from $\mathrm{DC}$ to $5 \mathrm{MHz}$ were observed. Furthermore, an optical frequency domain range using the frequency-shifted feedback fiber laser with the tandem AOM was demonstrated. The linear relationships between the beat frequency change and the optical path difference in the Mach-Zehnder interferometer with three slopes were obtained by combining the upward and downward frequency shifts in the first and second SAWs.

\section{Waveguide-type AOM for RGB laser lights}

Figure 9 shows the measured optical spectral transmittance of the diffracted light from the single waveguidetype AOM using an LED source as an input light [5]. The property for a bulk-type AOM fabricated in $\mathrm{TeO}_{2}$ with a driving frequency of $80 \mathrm{MHz}$ is also shown in the same figure. From these results, it was found that

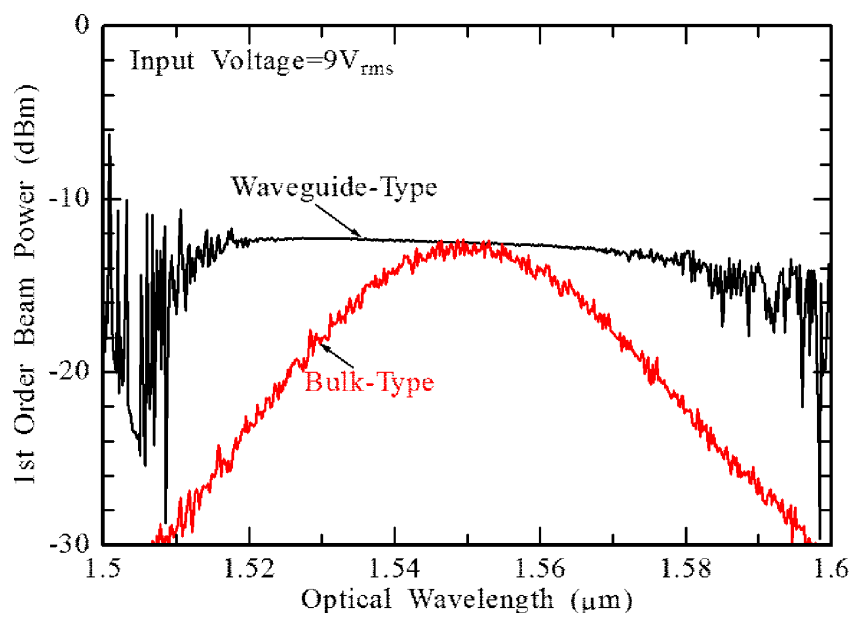

Fig. 9. Measured optical transmittance of the diffracted light using a LED source [5]. 
the waveguide-type AOM can perform modulation in a wider optical wavelength range than a bulk-type AOM at the same driving frequency. This is because the Bragg condition is relaxed due to the tapered waveguide.

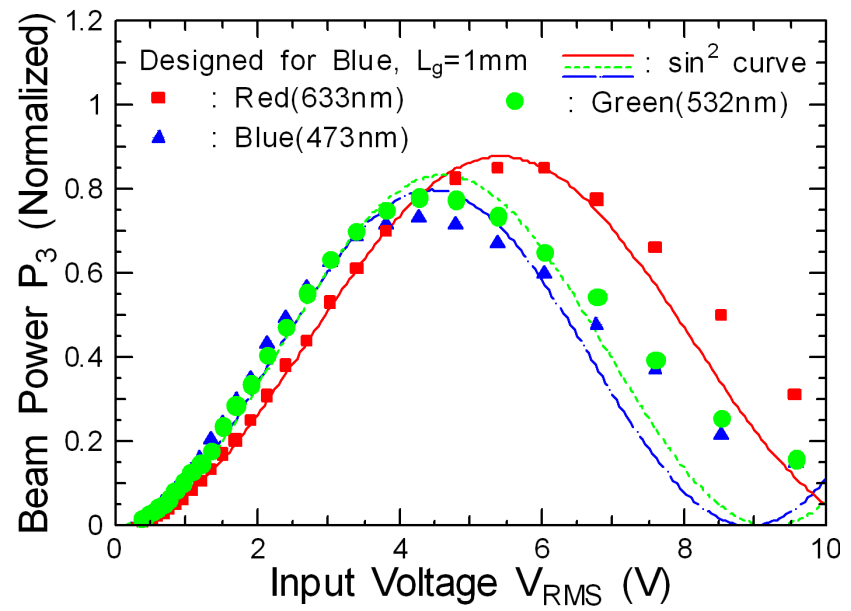

Fig. 10. Diffraction properties of RGB AOM [11].

This feature was applied to the visible range and realized an AOM that can modulate laser lights of the three primary colors red, green, and blue by the same modulator at the same driving frequency $[11,12]$. The configuration is similar to the waveguide-type AOM for $1.55 \mu \mathrm{m}$. The IDT with $\Lambda=16 \mu \mathrm{m}$ was adopted. The RGB laser lights were modulated with diffraction efficiencies of about 70 or $80 \%$ and a driving SAW power of less than $0.2 \mathrm{~W}$ at a driving frequency of $245 \mathrm{MHz}$, as shown in Fig. 10. By combining the AOM, laser sources, WDM couplers, and fiber delay lines a simple system for the simultaneous modulation of RGB laser was proposed, and the modulated light with a switching time of about $30 \mathrm{~ns}$ was demonstrated. The system is also in trial production in the cooperative company.

\section{Conclusions}

In this paper, the SAW mode dependence in the $\mathrm{AO}$ Bragg diffraction was discussed, and performances of several devices that the author and the coworkers developed were reviewed. It was confirmed that a suitable combination for obtaining a lower driving power is the TM mode with a Rayleigh wave. On the basis of the condition, a waveguide-type AOM in a tapered crossed-channel waveguide for an optical wavelength of $1.55 \mu \mathrm{m}$ was proposed. A tandem AOM was also proposed and doubly diffracted efficiency of $63 \%$ was obtained. Furthermore, an RGBAOM with about 70 or $80 \%$ diffraction efficiency was also fabricated at the same driving frequency.

\section{References}

[1] Y. Ohmachi, J. Appl. Phys. 44, 3928 (1973).

[2] C.S. Tsai, C.T. Lee, C.C. Lee, IEEE Ultrason. Symp. 1982, 422 (1982).

[3] R.W. Dixon, J. Appl. Phys. 38, 5149 (1967).

[4] S. Kakio, N. Zou, M. Kitamura, H. Ito, Y. Nakagawa, Jpn. J. Appl. Phys. 42, 3063 (2003).

[5] S. Kakio, M. Kitamura, Y. Nakagawa, N. Zou, T. Hara, H. Ito, T. Iizuka, T. Kobayashi, M. Watanabe, IEICE Trans. J86-C, 1263 (2003) [in Japanese].

[6] S. Kakio, S. Uotani, Y. Nakagawa, T. Hara, H. Ito, T. Iizuka, T. Kobayashi, M. Watanabe, Jpn. J. Appl. Phys. 44, 4472 (2005).

[7] S. Kakio, S. Uotani, Y. Nakagawa, T. Hara, H. Ito, T. Kobayashi, M. Watanabe, Jpn. J. Appl. Phys. 46, 669 (2007).

[8] S. Kakio, S. Uotani, M. Kitamura, Y. Nakagawa, T. Hara, H. Ito, T. Kobayashi, M. Watanabe, Jpn. J. Appl. Phys. 46, 4608 (2007).

[9] S. Kakio, M. Kitamura, Y. Nakagawa, T. Hara, H. Ito, Jpn. J. Appl. Phys. 47, 3992 (2008).

[10] S. Kakio, S. Shinkai, Y. Nakagawa, T. Hara, H. Ito, Jpn. J. Appl. Phys. 48, $07 \mathrm{GE07}$ (2009).

[11] S. Kakio, S. Shinkai, H. Kawate, Y. Nakagawa, Jpn. J. Appl. Phys. 48, 07GE02 (2009).

[12] S. Kakio, S. Shinkai, Y. Nakagawa, Jpn. J. Appl. Phys. 49, 07HD18 (2010).

[13] K.W. Loh, W.S.C. Chang, W.R. Smith, T. Grudkowski, Appl. Opt. 15, 156 (1976).

[14] C.S. Tsai, IEEE Trans. Circuits Syst. CAS-26, 1072 (1979).

[15] Guided-Wave Acoustooptic Bragg Interactions, Devices, and Applications, Ed. C.S. Tsai, SpringerVerlag, Berlin 1990.

[16] K. Yamanouchi, K. Shibayama, J. Appl. Phys. 43, 856 (1972).

[17] S. Kakio, S. Satoh, Jpn. J. Appl. Phys. 51, 07GC12 (2012).

[18] C.S. Tsai, IEEE Trans. Ultrason. Ferroel. Freq. Control 39, 529 (1992).

[19] Z.Y. Cheng, C.S. Tsai, Appl. Phys. Lett. 60, 12 (1992).

[20] A.K. Roy, C.S. Tsai, IEEE Photon. Technol. Lett. 4, 731 (1992). 